Rev. Chil. Pediatr. 65 (3); 161-165, 1994

\title{
Hemocromatosis perinatal
}

\author{
María Eugenia Hubner G.'; Rosemarie Bentjerodt R.,; \\ Rodrigo Ramírez F. '; Julio Nazer $\mathrm{H}^{1}{ }^{1}$
}

\section{Hemochromatosis in a newborn infant}

\begin{abstract}
A male newborn infanl with severe pulmonary valve slenosis, bicuspid oortic volve, ventriculor septal defect, horse shoe malformation of the kidney, toe polydactwia and syndoctylia together with persistent conjugated hyperbilirubinemla, low prolhrombin time, edema, hypaproleinemia since the first lew days of life is described. Frostaglandin $E$ infussion was necessary in the first hours of life and a moditied Blalock-Taussig shunt procedure was performed at age 5 days becouse of repealed episodes of cianosis, apnea and bradycardia. Cardiovascular stability was obtained from then on, but his clinical course was marked by progresive hepatic faiture, encefalopalhy, and renol insuliciency. The infant died on doy 28 of life. Past mortem examinalion showed difuse hapalic fibrosis and iron deposits were identified in his lungs, liver, pancreas, thyorid, gastrointestinal traci and salivary giands by prussian blue hystochemic slains. This lindings are characteristic of perinatal hemochrematosis wich is a lethal disorder rarely diagnosed before death.
\end{abstract}

|Key words: hemochromatosis, congenilal obncrmalites, birth defecls, newborn.;

La colestasia neonatal implica un diagnóstico diferencial complejo entre numerosas causas, varias de las cuales evolucionan hacia la cirrosis o una enfermedad hepática terminal en los primeros meses de vida. Una de ellas es la hemocromatosis perinatal, caracterizada por depósitos anómalos de fierro producidos durante cl desatrollo intrauterino, que ocurren en el hígado y otros órganos del feto ${ }^{1,2}$.

Con el propósito de estimular el interếs en la identificación del trastorno y el estudio de su naturaleza y manejo, se describen la forma de presentación, el diagnóstico diferencial, la anatomía patológica y las teorias sobre la patogenia de esta enfermedad a propósito de un paciente afectado por ella.

\section{Caso clínico}

Varón. Nacido de madre gesta 7, para 5, dos abortos espontáneos de primer trimestre cuya causa no fue estudia-

1. Servicio de Neonatología, Depantamento de Obstelricia y Ginecología, Hospital Clínico Umiversidad de Chile.

2. Servicio de Anatomía Patológica, Hospital Clínico Universidad de Chile. da, de 42 años de edad. Polihidroamnios. La madre, previameme sana, curs 6 desde las 34 semanas de gestacion con diabetes. Padre sano de 51 años, no consanguíneo. Ambos progenitores sin antecedenles de exposiciôn a drogas, alcohol, infecciones ni teratógenos. Parto vaginal a las 38 semanas con fórceps por sufrimiento fetal agudo. Peso de nacimiento $3770 \mathrm{~g}$, talla $52 \mathrm{~cm}$. Apgar $1 \mathrm{~min}=2,5 \mathrm{~min}=7$ y 10 $\min =7$. Desde el nacimiento mosto cianosis generalizada, mantenida y taquicardia. Nariz butbosa con narinas antevertidas, micrognatia, hipoplașia de ambas masnilas, soplo card faco sisuolico 2/6. Tres $\mathrm{cm}$ bajo el borde costal derecho se palpaba límile inferior del higado y en el borde costal izquierdo el polo inferior de bazo. Arteria umbilical única. Polidactilia posaxial con dos falanges y uñas en ambas maros. Sind actilia membranosa entre cuarto y quinto ortejos derechos; polidactilia y sindactilia membranosa parcial entre segundo y terccro y tercero y cuarto ottejos izquierdos. Ingresó a la unidad de cuidados intensivos con signos de insuficiencia cardíaca, sicndo tratado con oxígeno en capota $\left(\mathrm{FiO}_{2}\right.$ de 0,30$)$ solución de glucosa $10 \%$ iv $60 \mathrm{ml} \cdot \mathrm{kg}$. pero cursó con hipotensión, taquicardia y caída persisiente de la saturación con oxígeno de la sangre arterial, por lo que fue necesario aumentar la $\mathrm{FiO}_{2}$ a 0,50 e indicar dopamina $0.5 \cdot \mu \mathrm{g} \cdot \mathrm{kg} \cdot \min$. A las 17 horas de vida, después de identificar -por ulirasonografia- estenosis pulmonar seve$r a$, defecto septal interventricular perimembranoso y ductus arterioso penneable, se suspendio el oxígeno e inicío infusión de prostaglandina $E_{\text {, iv }} 0,5 \mathrm{mg} \cdot \mathrm{kg} \cdot \mathrm{min}$. No obstante, a las 20 boras de vida sufrío una crisis de hipertonía muscular y paro respiraterio, por lo que se conect $\delta$ a ventilador mecánico y se disminuyo la infusión de prostaglandina $\mathbf{E}$ a $0,01 \mathrm{mg} \cdot \mathrm{kg} \cdot \mathrm{min}$, pudiendo ser retírado del veatilador 28 horas más tarde, en condiciones hemodinámicas satisfactorias (presión venosa central $9 \mathrm{~cm}$ de agua, presión arterial 
media $57 \mathrm{~mm} \mathrm{II}_{\mathrm{g}}$ diuresis adecuada). Sin embargo fue nece. sario suministrarle plasma iresco y vitamina $\mathrm{K}$ por hipoprotromtinemia exlsema (tjempo dc protrombina indefinidamente prolongado) y, a la edad de tres días y medio, reinsta]ar nuevamente en ventilador por una tercera crisis de hipertonía, paro rcspiratorio y bradicardia extrema, con hemalocrito $56 \%$, glicenia $35 \mathrm{mg} \%$, hipoxemia arterial leve, acidosis mixta. En la ultrasonografía abdominal se regisıó riñón cn herradura de estructura nomal y gran hemorragia suprarrenal derecha. A la edad de cinco días se realizb una conexión arterial entre la aura y la artena pulmonar mediante el procedimiento de Blalock-Taussing modificado; a partir de entonces cursó sin mayores problemas en la función cardiovascular. Sin cmbargo evolucionó con hipoglicemia (a pesar de aumento del aporte intravenoso de glucosa a $6 \mathrm{mg} \cdot \mathrm{kg}$ - min); hipoprotrombinemia; hiperilitrubinemia (hasta $18,4 \mathrm{mg} / \mathrm{dl}$ ); fosfatasas alcalinas y transamnasas elcvadas (oxaloacética $120 \mathrm{U} /$ y pirúvica $108 \mathrm{~L}$ h a los 11 dias de vida); hipocalemia; trombocitopenia (63 000 plaquelas $/ \mathrm{mm}^{3}$ ), cdèna, que atumettó cápidamente: insuficiciciá hepática scvera y progrcsiva; encefalopatía e insuliciencia renal, falleciendo a los 28 días, con falla multisistémica. Fl paciente recibió cinco Iransfusiones de glóbulos rojos desde el sexto día devida, todas cllas después de la detección de signos clínicos y de laboratorio de insuficiencia hepática. Las pruebas scrolúgicas para toxoplas. mosis y rubcola dicren resultados negativos en la madre y el recién nacido. Fil carítipo del niño no mostraba alteraciones cromosónicas.

In la necropsia había edema generalizado, el peso corporal cra $5 \mathrm{~kg}$, tenía ictericia scvera, hidertórax bilateral y ascitis leve. El cocéfalo pesaba $360 \mathrm{~g}$ y había infartos venosos, hemortágicos y múltiples en la sustancia blanca de ambos hemisferios. El corazón pesaba $68 \mathrm{~g}$ (más del doble de lo nomal), había escenosis pulmonar severa, la válvula sorla era bicúspide, sc cricontró una comunicación interventricular membranosa puntiforme y el ductus arterioso cstaba ampliamente abierto. Habia hipertrofia miocárdica global, principalmente en el ventrículo derecho -cuyo grosor era de $13 \mathrm{~mm}-$, y varios infartos en la pared lateral del ventrículo izquierdo, de hasıa $15 \mathrm{~mm}$ de diámeıro. Ambos pulmones pesaron $67 \mathrm{~g}$; en el examen microscópicu se observaba en ellos cdema focal, hemoragias recientes y numerosos his. tiocitos intraalveolarcs cargados de pigmento pardo-amarillento (ligura I). El hígado era de tamuño nomal, verdoso. firme sin nódulos y las vís biliares éan permeables. La estrucura lobulillar estaba distorsionada por tractos fibrosos con conductillos biliares neofornados; habia colestasia, estatusis leve y múluiples depósilos de un pigmento marrón en los hepalucitos y el epitelio biliar (figura 2). Fin el riñón, conformado en herradura y de $63 \mathrm{~g} \mathrm{de} \mathrm{peso,} \mathrm{se} \mathrm{cn-}$ contró nccrosis ubular focal y nefrosis colémica acentuada. Ein la gláridula suprarrenal dcrecha, de $4.5 \mathrm{~cm}$ de tamaño, se obscrvaba necrosis hcjnorágica masiva. El timo tenúa aspeclo involutivo.

Con tincion de azul de Prusia se identificaron múliples depósitos de fierro en los macrófagos pulmonares, las células del parénquima hepálico y en cantidades menoresen los acinos pancreálicos, las cêlulas foliculares ủel tiroides. los rübulos tenales, las glándulas salivales menores y el epitelio sastrointestinal. En cl bazo y la médula osea se encontraron depósitos ucasionales del mismo tipo en las células ơl sistenta de monocitos macrólagos (ex sistema réículo endotelial). L a tinción PAS-diastasa de los hepatocíos no mustró lus glóbulos cosinofílicos característicos de1 déficil de alfa $!$ antitripsina.

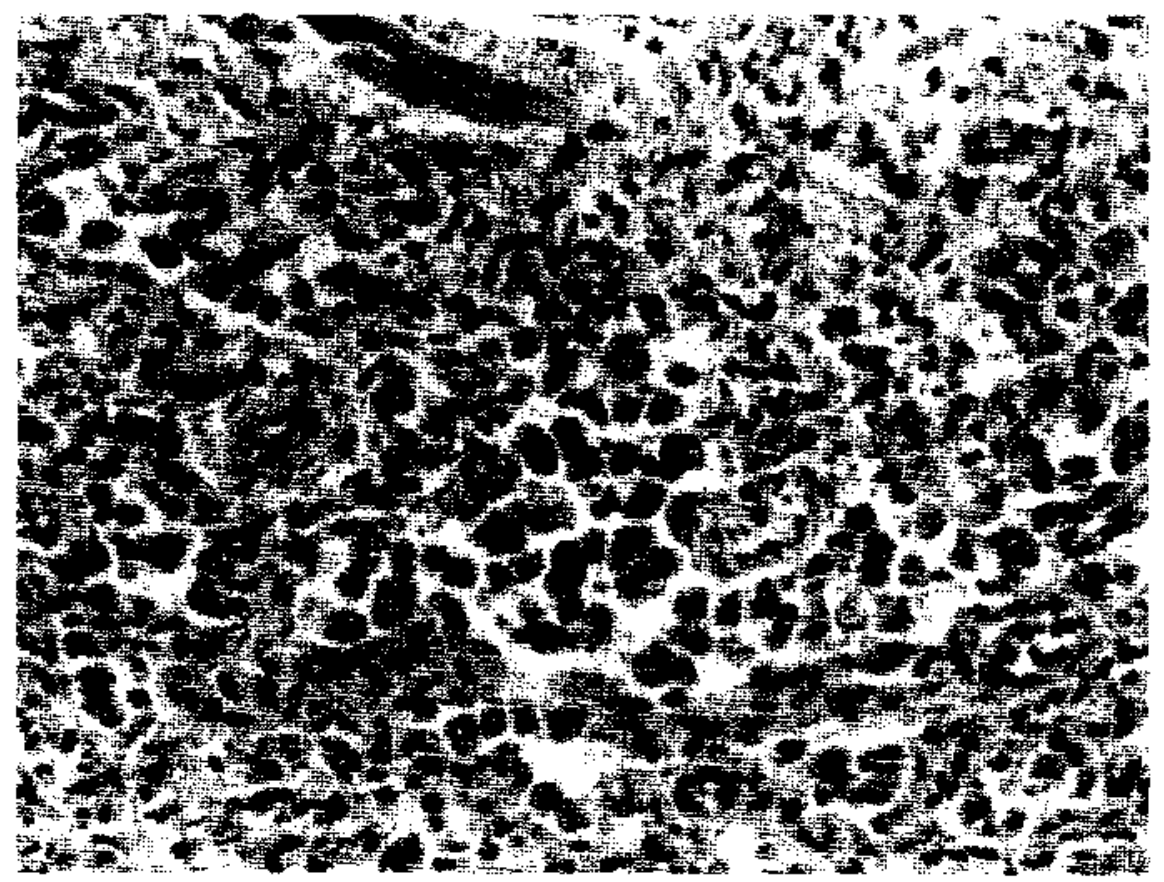

Figara 1: Corte de pulmón con numeroses histiocitos alveolares (tinción hematoxilina-cosina, $125 \mathrm{x}$ ). 


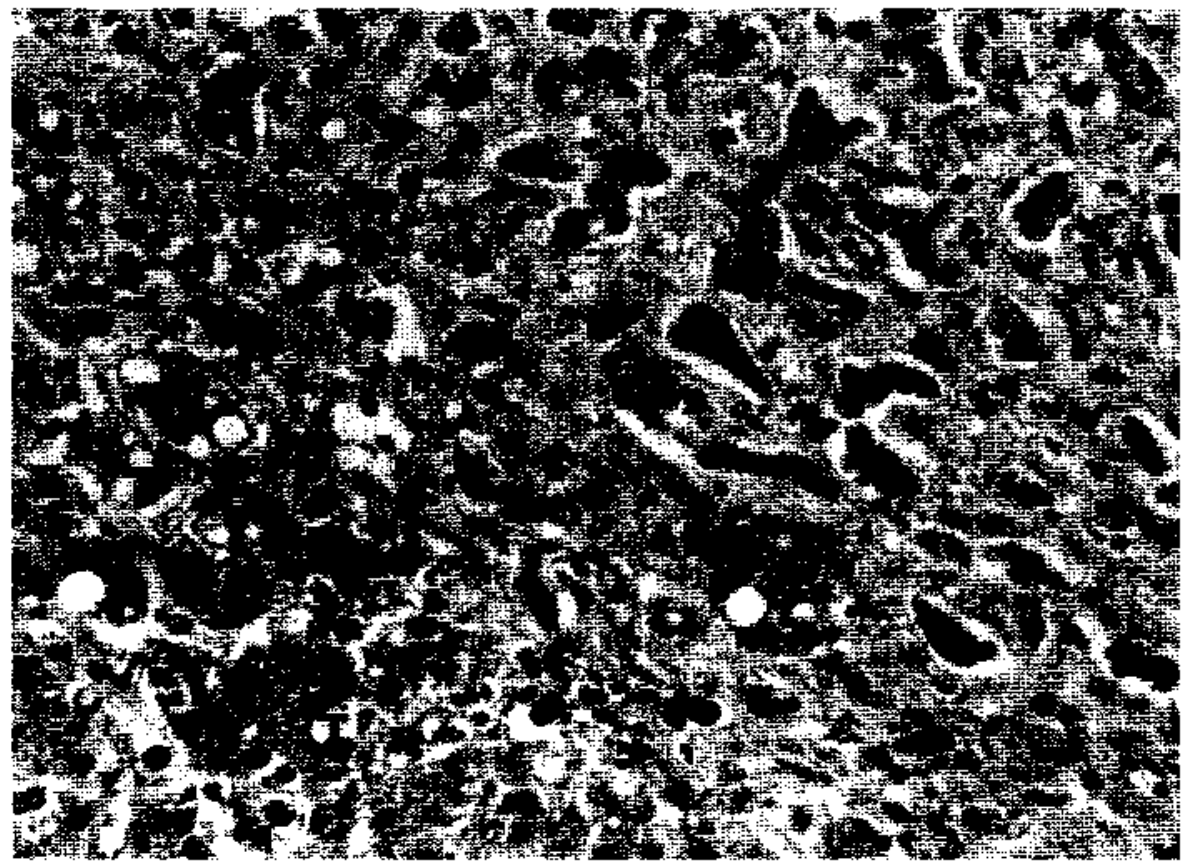

Figura 2: Corte de hígado con esteatosis leve, fibrosis con colangiolos neofornados y depósitos de pigmento en hepatocitos y cpilclio biliar (tinción hematoxjlina-cosina, $125 \mathrm{x}$ ).

\section{Comentario}

La hemocromatosis perinatal fue descrita por primera vez por Cattier en 1987 y desde esa fecha se han publicado 37 casos debidamente documentados. Su incidencia parece ser rclativamente baja entre olras causas de hepatitis colestásica neonatal, en comparación con la de hepatitis idiopática neonatal (1:4 800); la atresia de vías biliares (1: 12000$)$ y la deficiencia de alfa antitripsina (1:19 200). La verdadera incidencia de hemocromatosis perinatal podria ser más alta, porque puede haber muchos casos no comunicados, ya que rara vez el diagnóstico se confirma antes de la mucrte del niño y si no sc realizan la necropsia y las inciones histoquímicas adccuadas $^{3.6}$. Ambos sexos están igualmente representados en los casos conocidos. En 87\% de cllos los síntomas comenzaron el primer día de vida y, generalmente, se trataba de niños muy enfermos al nacer, que sufrieron edema y anasarca, diátesis hemorrágica, ictericia progresivamente mayor. Todas estas expresiones de una cnfermedad hepática severa, rápido deterioro orgánico y mucrte, también ocurrieron en el niño que se comenta. Once de 37 casos descritos ante- riormente fallecicron al segundo día de vida, siendo los márgenes de edad, al morir, uno y cicnto veintidós días. La concentración de transaminasas en el suero ha sido frecuentemente normal o ligeramente elevada, como en este paciente. La hipoprotrombinemia, constante en la evolución del recién nacido aqui descrito, se ha registrado en todos los casos conocidos, asi como la hipoproteincmia -hallazgo característico, responsable al menos en parte del edema-, la hiperbilirrubinemia de predominio directo y las fosfatasas alcalinas discretamente clevadas, que también fueron anotadas en este recién nacido ${ }^{4}$.

Los hallazgos histológicos, esenciales para el diagnóstico de hemocromatosis perinatal, consisten en depósitos masivos de fierro en los hepatocitos -principalmente los de ubicación periportal-, como lámbién en el epitelio de los conductos biliares y, además, otros de igual naturaleza, pero de cuantía variable, en numerosos parénquimas: miocardio, páncreas, tiroides, suprarrenales, tracto gastrointestinal, paratiroides, glándulas ecrinas, salivales, plexos coroideos, endometrio, mama, tesliculos ${ }^{2}$, hipofisis y timo. Los acopios de fierro son escasos o no existen en las células del sistema de los monocitos macrófagos (ex 
reticulo endotelial) del bazo y médula ósea. En el hígado se encuentra necrosis hepatocelular, $\mathrm{co}$ lapso reticular, librosis, proliferación de conductillos biliares y colestasis. En algunas ocasiones se agregan, a los hallazgos recién descritos, transformación en células gigantes, esteatosis mínima, ectasia linfática portal y esclerosis de venas centrolobulillares. En el páncreas sc describe hipertrofia e hiperplasia de los islotes y en el riñón, dilatación tubular ocasional y nefromegalia ${ }^{4}$. El diagnóstico diferencial debc plantearse con el sindrome de Zellweger, la tirosinemin, cl déficit đe $\alpha 1$ antitripsina, toxoplasmosis, rubéola, trisomía 22 y hemosiderosis postransfusional ${ }^{4}$. En cl riñón de nuestro paciente no se encontraron los quistes característicos del síndrome de Zellweger y de la tirosinemia; en esta última la esteatosis y la formación de vacuolas citoplasmáticas son, además, muy prominentes en los hepatocitos, pero en este caso ambos fenómenos eran mínimos. La tinción de PAS con diastasa no revelo los glóbulos cosinófilos hepatocitarios característicos del déficit de alfa $l$ antitripsina. El cariograma normal desecha en csie caso la posibilidad de una trisomía 22 y no había signos clínicos y scrológicos de nubćola o toxoplasmosis en la madre y cl niño. La hemosiderosis secundaria a transfusiones puede ser descartada, pues las manifestaciones clínicas y las prucbas de laboratorio sugerían insuficiencia hepática previa a las tranfusiones que recibió este niño en las últimas fases de su enfermedad y porque sólo habia ocasionales depósitos de fierro en las cćlulas del sistema de los monocitos macrófagos.

Lá patogénesis de la hemocromatosis perinatal es poco clara. La hemocromatosis del adulto es un desorden autosómico rccesivo y se asocia estrechamente con los antígenos de histocompatibilidad HLA tipos $A 3, B 7$ y $B 14^{8}$. El análisis de esta asociación ha permitido dilucidar el modelo genético de transmisión de esa enfermedad, en el que está implicado un gen recesivo ligado al HLA y ubicado en el brazo corto del cromosoma 6. El haplotipo HLA A3 se ha asociado a hemocromatosis idiopática del adulto en $68,7 \%$ de $\operatorname{los} \operatorname{casos}^{6,8}$. Sin embargo, HLA A.3 no parce asociado con hemocromatosis neonatal en un estudio de varios afectados y sus familias ${ }^{9}$. Nuestro paciente presentaba varias malformaciones significativas, con cariotipo normal y no tenemos explicación para cllas, pucs en los casos registrados anteriormente no se había descrito asocia- ción con malformaciones congénitas, con excepción de un niño que sufría de atresia esofágica ${ }^{10}$.

Se dcsconoce cuál es el defecto básico responsable del aumento del contenido de fícro celular en la bemocromatosis neonatal. Tampoco se sabe si el trastorno está determinado genélicamentc, resultando en un excesivo transporte de fierro a través de la placenta, o si su causa es una redistribución anómala de los depósitos de lierro. Los mecanismos responsables de la injuria hepatocelular tampoco están claros. Se ha sugerido que un agente transmisible de la madre al foto (por ejemplo un virus) podría causar daño hepatocelular y secundariamente derivar en una anormalidad en el metabolismo del fierro ${ }^{1 !}$.

Crcenos, con otros autores, que para comprender mejor estos procesos y mancjarlos de manera más adecuada es fundamental hacer estudios histopatológicos completos, icluyendo técnicas histoquímicas de azul de Prusia, en el tejido hepático de todos los reciến nacidos con colestasia cuya causa no ha sido determinada.

\section{Resumen}

Se describe un recién nacido afectado por varias matiormaciones cardiovasculares (estenosis valvular pulmonar severa, defecto septal intervenuricular, aorta bicúspide), riñón en herradura, polidactilia y sindactilia de los ortejos, quicn presentó muy precozmente hiperbilirrubinemia de prodominio directo, cdema, hipoprotrombinemia refractaria a la vitamina $\mathrm{K}$ e bipoproteinemia. Después de suministrarle prostaglandina $E$ en las primeras horas de vida y realizar un procedimiento cardiovascular paliativo (anastomosis de arteria sistémica a arteria pulmonar de BlalockTaussig), con el que ccdicron las crisis de apnca y bradicardia que le afectaban, el paciente evolucionó con insulicicncia hepática severa, encefaJopalía c insuficiencia renal y falleció a la edad de 28 dias. En la nccropsia se encontró fibrosis hepática, proliferación ductal y -con tinción histoquímica de azul de Prusia-múltiples depósitos de fierro en hígado, páncreas, pulmón, tiroides, tracto gastrointestinal y glándulas salivales. Estos hallazgos son característicos de la hemocromatosis perinatal, afección colestásica poco frecuentc y de alta letalidad que debe tenerse presente cn recién nacidos con insuficiencia hepática precoz y mantenida. La hemocromatosis 
neonatal no había sido descrita en asociación con malformaciones congénitas significativas, excepto por un caso con atresia esofágica.

(Palabras clave: hemocromatosis, recién nacido, malformacioncs congénitas.)

\section{Referencias}

1. Witzleben $C$, UriA: Perinatal hemochromatosis: entity or end resuli? Hum Pathol 1985; 20: 335-340.

2. Witzleben C, Uri A: Perinatal hemochromatosis: one disease, several disease or a spectrum? Hepatology $1990 ; 12: 176-177$.

3. Sptnok $R$, Grand $R$ : General configuration of cholestasis in the newbom. Semin Liver Dis 1983; 2: 381 392.

4. Harnard J, Manci E: Idiopathic neonalal ironstorage discase. Gastrocnterology 1991: 101: 1420 . 1427.
5. Blisard $K$, Barlow $S$ : Neonatal hemochromatosis, clinical, morphologic and quantilative iron studics. Pathol 1986; $17 \div 376-383$.

6. Silver $M$, Beverley $D$, Valberg $L$, Luz $E$, Phillips $M$, Shaheed $w$ : Perinatal heroochromatosis, clinical, morphologic and quantitative iron studies. Am J Pathol 1987; 128: 538-554.

7. Kurnetz R, Yang S, Holmes R: Neonatal jaundice and coagulopathy. J Pediatr 1985; 107: 982-987.

8. Escobar G, Heyman M. Smith W y Thales M: Primary hemochromatosis in childhood. Pedialrics 1987; 80: 549-554.

9. Hardy L, Hanser J, Kushner J, Knisefy A: Neonatal hemochromatosis: genetic analysis of transferrin-receptor, $\mathbf{H}$-apoferritin, and $\mathrm{L}$-a poferritin loci and of the human leukacyte antigen class 1 region. Am $J$ Pathol 1990: 137: 149-152.

10. Halliday J: Inheriled iron overload. Acta Paediatr Scand (Suppl) 1989: 361: 86-95.

11. Hoogstraten J, DerekJ. Knisely A. Fetal liver disease may precede extrahepatic siderosis in neonatal hemochromatosis. Gastroenterology 1990; 98: 1699-1701. 